\title{
THE SUBMILLIMETER-WAVE ASTRONOMY SATELLITE: ON-ORBIT THERMAL PERFORMANCE AND DESIGN RETROSPECTIVE
}

\author{
David A. Boyd \\ Smithsonian Astrophysical Observatory
}

Wes Ousley and Louis Fantano

NASA Goddard Spaceflight Center

\section{ABSTRACT}

A large telescope aperture, stringent thermal stability and temperature range requirements, and a passivelycooled $150^{\circ} \mathrm{K}$ module presented major challenges in thermal design and hardware fabrication of this Small Explorer satellite. This paper reviews briefly the thermal design of the SWAS science instrument, and examines the first three months of on-orbit thermal history. Measured temperatures for both the science payload and the spacecraft module and solar arrays are compared with those predicted by the correlated analytical model. Similarities and differences are interpreted in terms of the major uncertainties remaining after thermal-balance testing, especially those of MLI performance and telescope aperture properties. Review of the thermal model adequacy and thermal design verification are included to suggest improvements in the thermal design process for future missions.

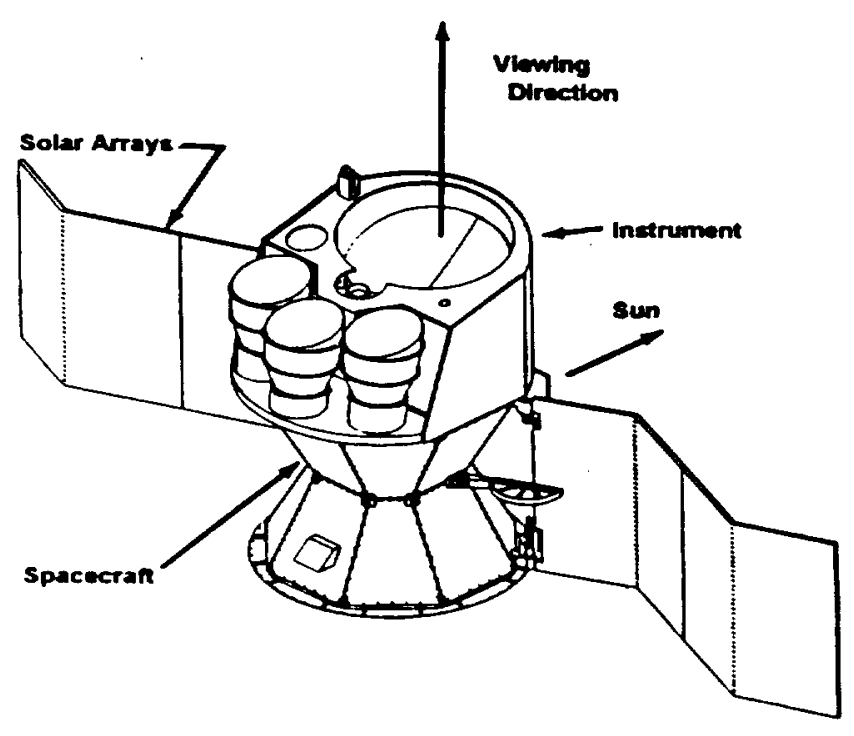

Figure 1: The Submillimeter-Wave Astronomy Satellite

\section{INTRODUCTION}

The Submillimeter Wave Astronomy Satellite (SWAS), shown in Figure 1, was launched successfully on Dec. 5, 1998 , aboard a Pegasus XL vehicle, into a $650 \mathrm{~km}$ near circular orbit at 67 degrees inclination. SWAS is a SmallExplorer class radiotelescope for observation of emissions around $500 \mathrm{GHz}$ from star-formation regions. It has a folded off-axis $\mathrm{f}-4$ Cassegrain optical train with 0.3square-meter aperture, feeding the $150^{\circ} \mathrm{K}$ first stage of a multi-module receiver system that modulates an Acousto-Optical Spectrometer. The telescope and receiver are packaged with a startracker and three radiative coolers in a half-cubic-meter envelope. Below the instrument is a Small Explorer (SMEX) spacecraft module that supports the solar arrays, and encloses the power, telemetry and attitude control systems.

\section{THERMAL SYSTEM DESIGN REQUIREMENTS}

Four fundamental characteristics of the instrument system dictated the choice of thermal design features: the $0.3 \mathrm{~m}^{2}$ open observing aperture, a receiver first stage with a $150^{\circ} \mathrm{K}$ desired operating temperature, a narrow $\left(20^{\circ} \mathrm{C}\right)$ allowable operating temperature range for the spectrometer, and a thermal stability requirement near $0.01^{\circ} \mathrm{C} /$ minute for parts of the receiver subsystem. Limits on available power in the worst end-of-life design case originally dictated a passive design without heaters and active control systems; passive control of a package of this size with only 45 watts of science power required high-performance multilayer insulation (MLI) everywhere except for viewing apertures and two radiator panels. These radiators were partly MLI-covered, but oversized to allow temperature level adjustment after thermal tests. Power limits also dictated passive cooling for the $150^{\circ} \mathrm{K}$ first stage, a challenge for a satellite with variable pointing direction in a $600 \mathrm{~km}$ near-earth orbit. Figure 2 illustrates the most important elements and thermal design features of the SWAS instrument. 


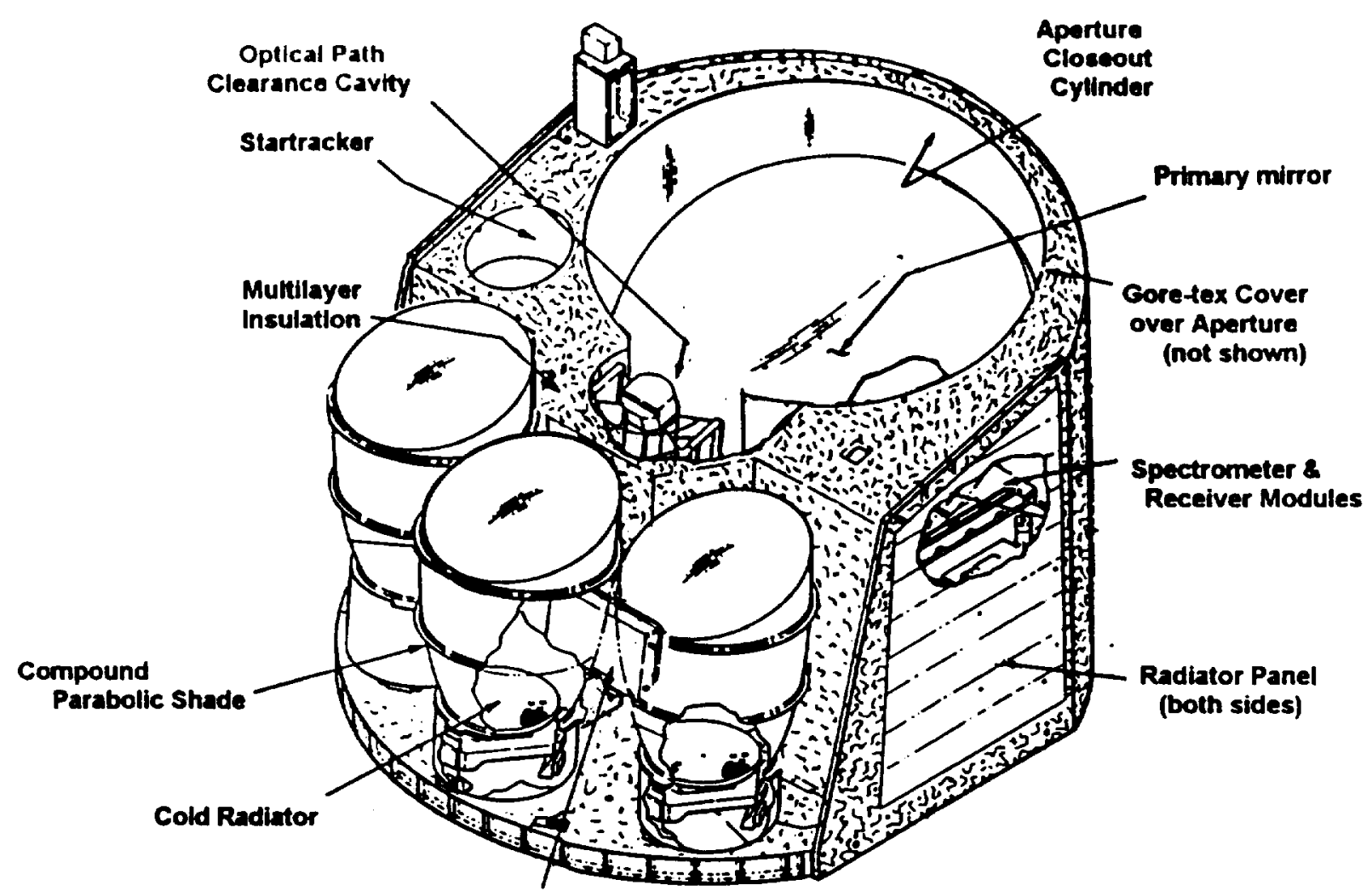

$160^{\circ} \mathrm{K}$ Receiver Module

Figure 2: Design Features of the SWAS Instrument

The 67-degree orbital inclination allows both full-sun and eclipsing orbits. Full sun is about $10^{\circ} \mathrm{C}$ warmer than maximum eclipse for the spacecraft, but the two cases are similar for the instrument because the sunward side is fully insulated. Science observation is confined to pointing the telescope within a $130^{\circ}$ cone about the local earth vertical while avoiding the $150^{\circ}$ cone centered on the sun, as illustrated in Figure 3. A typical observation points the telescope ahead to a rising pair of targets, one of which is a known empty calibration region. As they pass overhead and set behind, the spacecraft re-points the instrument at one-minute intervals to alternate between the two. A forward slew maneuver then points the telescope at the next target. Sun and earth avoidance are particularly critical at low beta angles near the subsolar point. The aperture is therefore mostly pointed away from the earth, greatly reducing effects of incident environmental radiation upon it.
Beta $=0^{\circ}$, Hot Case: Looking from Orbit Pole

View Direction: within $560^{\circ}$ from orbit plane, except near sun

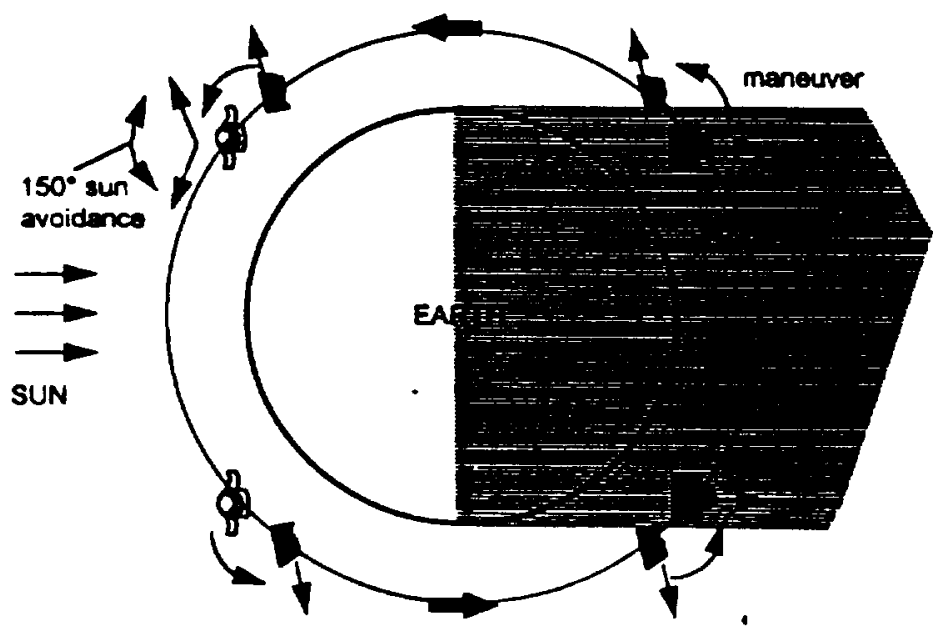

Beta $=90^{\circ}$, Cold Case: Looking from Sun View Direction in Orbit Plane

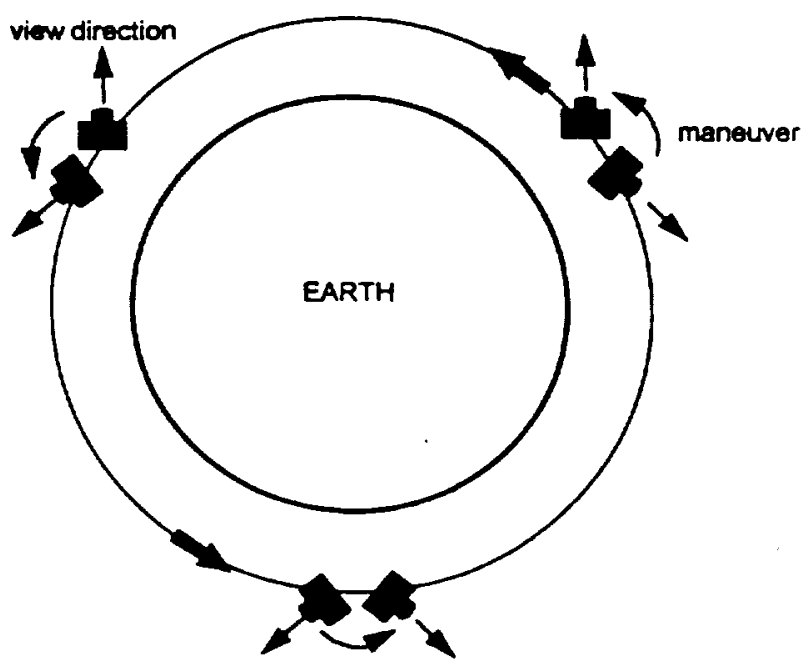

Figure 3: SWAS Viewing Attitudes in Hot and Cold Cases 
The instrument pointing profile presented thermal challenges to the spacecraft module design as well. Transient attitude-control power changes (75-135 watts) had to be accommodated in the design, and the frequent re-pointing greatly complicated environmental flux calculations. One solar array panel, $-40 /+90^{\circ} \mathrm{C}$ in fulleclipse orbits, was insulated from but mounted directly to the body of the spacecraft. Sun angle variations meant that most spacecraft surfaces had to accommodate direct solar exposure, including the launch vehicle adapter, where large areas were uncoated aluminum to ensure fail-safe launch separation.

\section{RECEIVER AND SPECTROMETER DESIGN}

To minimize thermal noise in the science data, a temperature of $150^{\circ} \mathrm{K}$ was the goal for the microwave receiver modules at the telescope focus and the first amplifier stages. The box containing these front-end components is supported by, and has low-conductance electrical and microwave connections to, other boxes near room temperature. A passive radiative cooler system was chosen, having three aluminum disc radiators with a combined area of $730 \mathrm{~cm}^{2}$. Radiating surfaces are honeycomb-faced for high emittance, and white-painted for albedo rejection. They are connected to the front-end box by flexible links of stacked aluminum foil strips. The entire cold system is radiatively isolated by $\mathrm{MLI}$ and mechanically supported by glass-epoxy composite flexures. The disc radiators are shielded from the incident planetary environment by Compound Parabolic[1] shades (CPS), which are designed for a 40degree halt-angle sharp cutoff for incident radiation. These shades are white outside for albedo rejection, and gold-plated inside for minimum thermal parasitic loss to the disc radiators located within. The CPS geometry differs from a conventional conical shade in several ways:

- it rejects completely all radiation entering the shade beyond a specific acceptance angle from the axis, but concentrates all radiation entering within the acceptance angle onto the exit aperture (the cold radiator surface)

- the acceptance angle cutoff is much sharper

- parasitic radiation from the shade interior can reach the radiator only by a direct path; all reflected radiation is rejected.

In spite of the great care with thermal isolation, parasitic losses into the cold module were predicted to exceed one watt (dominating the $100 \mathrm{mw}$ internal power), and measured much higher in test and during the mission.

The signal received at the cooled focal-plane unit is fed through RF amplifiers to the spectrometer, where it modulates the deflection of a laser diode beam by a crystal. The amplifiers, crystal and laser diode are all temperature-sensitive. Required thermal stability of the spectrometer and receiver subsystem is about $0.01^{\circ} \mathrm{C}$ over a typical 100-second observing sequence. Because of the large predicted temperature transients induced by the orbital cycle, insulating spacers were added to the mounting interface of critical boxes to increase their time constants and reduce lemperature swings.

\section{APERTURE REGION DESCRIPTION}

The instrument thermal balance is dominated by the $0.3 \mathrm{~m}^{2}$ telescope aperture, which has a potential emissive power of about 100 watts at instrument operating temperature. Effective thermal control depends on lowemittance surfaces, careful closeout between the mirror and instrument structure, and MLI locally where clearance around the folded optical path creates a radiating cavity. Uncertainty in the emittances and temperatures, especially of the large low-emittance surfaces, contributes significantly to the uncertainty in heat loss from this large area. The aperture cover, a form of Gore-tex@ Teflon nearly transparent to $500 \mathrm{GHz}$ radiation, has non-uniform infrared transmission with wavelength, and its transmission varies with the source temperature of radiation. This non-uniformity creates uncertainty both in modeling of the aperture and in thermal-balance testing.

The entire external surface of the instrument, excepting the thermal radiator surfaces, telescope and sensor apertures, and radiative cooler shades, is covered with 18-layer MLI. Construction is conventional with mesh internal spacers and Kapton outer layers, with an additional layer of silver-Teflon on the sun-facing side.

\section{INSTRUMENT THERMAL BALANCE TEST RESULTS}

Before instrument integration into the satellite, combined thermal-balance and functional thermal-vacuum tests were conducted. The goal of the thermal-balance test was verification and calibration of the instrument thermal model. From the outset of the test it was evident that the thermal model was not a good predictor of the test article's thermal configuration. The 47-watt instrument was expected to require only a few watts of test heater power to maintain minimum operating temperatures; in fact, about 50 watts additional power was needed. Thermal gradient patterns in the instrument pointed to a severe heat leak on the anti-sun side of the instrument.

After instrument-level thermal testing was completed, an inspection revealed several areas where the thermal model did not correspond to the hardware. Thermal blanket construction also differed from thermal model assumptions. Substantial thermal system modifications were required to allow on-orbit temperatures to slay above minimum limits, including keeping the spectrometer at a minimum of $5^{\circ} \mathrm{C}$ with sufficient thermal stability. The most important thermal changes incorporated after the test were complete replacement of external MLI blankets with larger pieces and with allowances for thermal contraction, and closeout of gaps allowing parasitic heat leaks [2]. Verification of the new thermal system required adding instrument thermal balance exposures to the integrated-satellite thermal vacuum test. 


\section{SATELLITE-LEVEL THERMAL TEST}

The main instrument thermal system goals for the satellite thermal balance test were to demonstrate the thermal performance of the modified instrument in the required operating temperature range, and to acquire data to correlate the thermal model. Heater selection and final radiator sizing were accomplished and orbital predictions made using the correlated model. (The radiators were left somewhat oversized for the test to allow positive thermal control of the instrument during all test phases.) Dominant heat losses were through the instrument aperture, radiators, and MLI blankets.

Telescope aperture heat loss was difficult to predict or verify by analysis because of the large area of curved polished aluminum surfaces and the spectrally-variable infrared transmittance of the Gore-tex cover. Two extra thermal balance cases were added to measure directly the aperture heat loss. Radiator effectiveness was tested by changing the temperatures of the test cold plates facing the radiators. The remainder of the heat loss was through the MLI blankets, and the heat loss summations produced a very reasonable $\mathrm{MLI}$ effective emittance value of 0.015 .

Thermal-vacuum test goals for the spacecraft included acquiring data for model correlation, verifying operational modes, and accumulating operating time at temperatures beyond tlight predictions. Conductive isolation from the solar arays was verified. and the launch vehicle adapter cousling to the spacecraft structure was characterized. $L: ?$ adiators dominated spacecraft heat balance, so !nLi effectiveness was not a major driver. No surprises were seen in spacecraft thermal data, and correlation with the spacecraft thermal model was routine.

\section{INSTRUMENT THERMAL MODEL CORRELATION AND ORBITAL PREDICTIONS}

It was eviden: from early test results that the reconfigured instrument with new MLI was close to test model predictions. Differences between the modelpredicted temperatures and test data at 44 locations averaged $1.5^{\circ} \mathrm{C}$ or less for all four thermal balance cases, with r.odel data biased higher than test data. The largest stancard deviation of the differences was $1.7^{\circ} \mathrm{C}$ in the cold operational case. However, uncertainties remained in predicted flight performance, arising from:

- post-test modification to the oversize radiators

- differences in MLI performance after blanket handling, reinstallation \& launch

- differences between test and orbit boundary conditions incident on the aperture and MLI.

Using the correlated model with reduced radiator area and orbital boundary conditions, the predicted hotcold range with allocations for uncertainties and degradation showed no margin. Furthermore, instrument attitudes determined by the science observation plan biased the mission at the cold end of the range most of the time. The spectrometer laser diode, the component with the most restrictive range, was predicted 10 operate about $10^{\circ} \mathrm{C}$ below its desirable lower limit in the cold case, and exhibited about $15^{\circ} \mathrm{C}$ margin in the hottest end-of-lite case. Heater/thermostat operations during test had degraded the science data quality, so ensuring the integrity of the cold-case instrument without temperature cycling was clearly the focus of final design trim. This concern was resolved by adding two independent heater circuits controlled by thermostats with close/open set points of $1 / 10^{\circ} \mathrm{C}$ and $10 / 20^{\circ} \mathrm{C}$. Because the overall instrument power sensitivity was about $1^{\circ} \mathrm{C} /$ watt, either five-watt heater, once enabled by its thermostat, would raise the steady state temperature by only $5^{\circ} \mathrm{C}$, not enough to open its thermostat and cycle in power. The net effect of the heaters was to cut about $10^{\circ} \mathrm{C}$ off the cold end of the predicted range, permitting a small cold bias to ensure margin in the hot case. Decreased radiator area, confirmed by the model to be half that of the test article, was accomplished by closing completely the radiator nearest the most stability-sensitive equipment, leaving the other radiator unchanged. This eliminated the orbital-period temperature cycle previously induced by that radiator surface.

\section{FINAL PREPARATIONS FOR LAUNCH}

SWAS was originally scheduled for launch in late 1995, but the launch was delayed because of launch vehicle performance difficulties. The satellite was stored in a controlled environment, with the largest MLI blanket stored separately. Its silver-Teflon outer layer was not installed before storage to minimize possible handling damage. The smaller closeout blankets were left in place on the instrument during storage.

The largest instrument blanket was installed just before shipping to the launch site. This blanket covers the sun facing surface and extends backward over the two side panels, with an opening for the experiment radiator surface. The outer silver Teflon layer covers only the sun-facing surface. It was installed over the outer layer by folding over the top and bottom edges, allowing plenty of slack for shrinkage with low temperatures, and securing it to the warm inner layer.

\section{LAUNCH AND EARLY-ORBIT THERMALCONDITIONS}

The satellite was launched successfully on Dec. 5, 1998, aboard a Pegasus XL vehicle, into a $650 \mathrm{~km}$ near-circular orbit at 67 degrees inclination. Orbital altitude achieved was within the expected range of altitude dispersion. The launch date resulted in an initial beta $=88 \mathrm{deg}$ solar orientation, with full sun for the first seven mission days. Initial satellite orientation was sun-pointed correctly with a slow roll about the satellite sun line, bringing the telescope aperture into periodic view of the earth. Spacecraft components, powered in operational mode. came to thermal equilibrium at an average of $2^{\circ} \mathrm{C}$ from predicted levels. The instrument, initially unpowered, cooled from a launch temperature of about $14^{\circ} \mathrm{C}$ to about $-10^{\circ} \mathrm{C}$ in 28 hours (Figure 4). Only two instrument 
temperatures were available in the satellite housekeeping data stream during the unpowered part of the mission. However, the iwo were not well-selected to represent the bulk thermal conditions, and the pre-launch verification had indicated discrepancies of as much as $3^{\circ} \mathrm{C}$ between the indicated lemperatures and measured conditions. Visibility into the actual cooling profile was therefore severely limited, and accuracy of the two reported temperatures was somewhat uncertain. Nevertheless, the two fivewatt platform heaters (six watts at the fullsun bus voltage) were observed to actuate near their set points of $10^{\circ} \mathrm{C}$ and $1{ }^{\circ} \mathrm{C}$, and turn-on of a small survival heater on an isolated electronics box was also observed.

The main survival heater was large enough by design to arrest the decreasing instrument temperature, and its actuation temperature was $9^{\circ} \mathrm{C}$ above the lower survival limit, a seemingly prudent margin. However, the temperature reporting uncertainty, combined with a long interval between ground-station contacts and somewhat unreliable ground station communication, caused us to turn the instrument on earlier than planned. The survival heater had not been tested since the environmental test three years earlier, and we were concerned that the instrument could reach hazardous temperatures if the heater failed to actuate and the next command access from a ground station was missed.

The instrument was powered by command before the main survival heater reached its actuation temperature, and temperatures immediately began to warm toward operating conditions (Figure 4). Furthermore, within a few hours the satellite attitude was stabilized in a mode facing either north or south with the aperture no longer viewing the earth directly, which slowed the temperature rise. By the end of the third mission day instrument temperatures were stabilized near $18^{\circ} \mathrm{C}$, and the first of the two platform heaters turned off at $10^{\circ} \mathrm{C}$. When the additional earth avoidance constraints of the science observations were established the instrument cooled slightly, but additional power during the early calibration observations caused an offsetting increase. Conditions were not stable enough to produce an unambiguous equilibrium, but most of the electronics appeared to be converging around $21-24^{\circ} \mathrm{C}$, and the instrument platform about $18^{\circ} \mathrm{C}$. The platform did not reach the $20^{\circ} \mathrm{C}$-shutoff temperature of the second platform heater, however.

Orbital precession resulted in an average beta-angle change of about 3.1 degrees/day, and eclipses began on the seventh mission day. With resulting decreases in spacecraft module intertace temperature and solar-array input, the instrument initially cooled at almost $2^{\circ} \mathrm{C} /$ day, and would probably have reached a minimum of $11-12^{\circ} \mathrm{C}$ except for additional intermittent power bursts. The power configuration finally stabilized after two weeks, and at that time the increased albedo was slowly

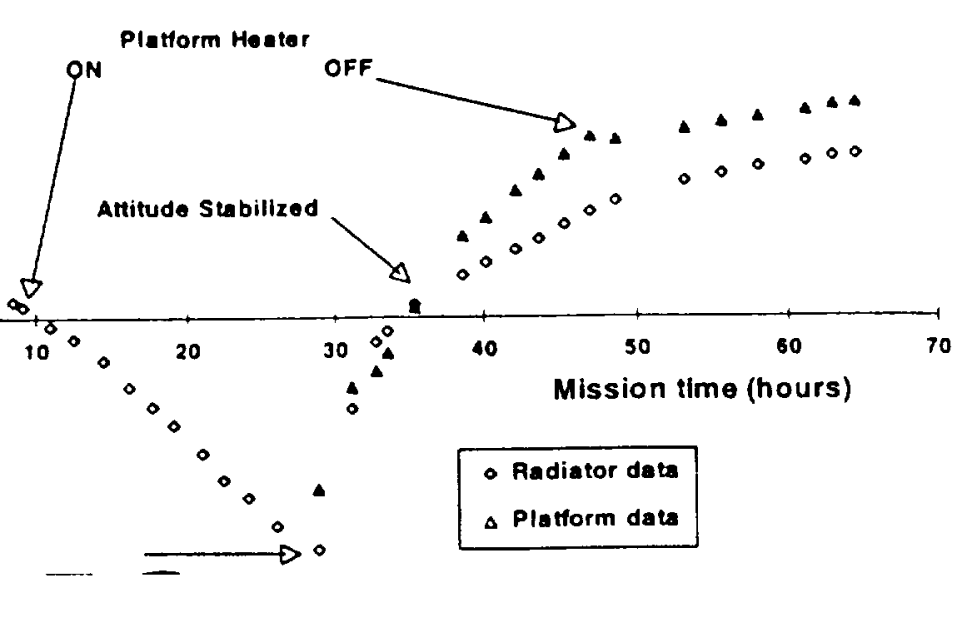

Figure 4: Measured Flight Data - flrst 3 mission days

producing warmer conditions. Temperatures leveled out on the 29th day of the mission (beta $=0$ ) about $3^{\circ} \mathrm{C}$ below their full-sun values, but also at about three watts less power with the advent of routine science observations.

\section{CORRELATION OF THE INSTRUMENT THERMAL MODEL WITH FLIGHT DATA}

Fourteen instrument temperatures are available in the flight data stream, but most of these are internal electronics temperatures useful primarily for state of health and troubleshooting. A single temperature on one of the three cold radiators serves as an indicator that the viewing constraints are being maintained. Three temperatures on the main instrument platform are available but are almost equal, and only the central temperature was chosen as a model correlation reference point. Bus voltage and total instrument bus current as well as individual subsystem bus currents are also available for power determination. An uncertainty of about $5 \%$ in total power is caused by discrepancy in total current versus the sum of the individual values; the second was used because the resolution is somewhat better. We observed total power to be about 2 watts above that measured in test, primarily because of higher startracker power in actual obsenvation modes rather than test modes.

The beta $=88^{\circ}$ December launch represents a warm but not extreme design case. The solar constant is high, but average albedo is expected to be low and the earth thermal emission above median because of the oceandominated daytime orbit track. The correlated thermal model with nominal parameters, as-measured on-orbit power and winter environmental boundary conditions predicts key instrument temperatures about $2^{\circ} \mathrm{C}$ below the quasi-equilibrium value on mission day 3 . Modifying the $E^{*}$ of the $M L I$ for the expected improved performance in zero-G after expansion by the trapped air during launch venting (corresponding to $E^{*}=0.012$ ) improves the correlation. Spacecraft temperatures were also within about $2^{\circ} \mathrm{C}$ of predictions at beta near 90 , with a $4^{\circ} \mathrm{C}$ difference at beta $=0$. (Table 1 ). 
Table 1: Measured vs. Predicted Temperatures (Celsius)

\begin{tabular}{|c|c|c|c|c|}
\hline \multicolumn{2}{|c|}{ Beta $=90$} & \multicolumn{2}{c|}{ Beta=0 } \\
\hline Flight & Model & Flight & Model & Location: \\
\hline 19 & 19 & 16 & 21 & Instrument Platform \\
\hline 22 & 22 & 19 & 24 & Spectrometer \\
\hline 24 & 22 & 21 & 24 & Microwave IF amp \\
\hline 23 & 22 & 10 & 16 & Spacecraft deck \\
\hline 17 & 16 & 7 & 9 & S/C computer \\
\hline 92 & 97 & 50 & 59 & Body-mounted array \\
\hline
\end{tabular}

As previously noted, the design-phase thermal model consistently indicated the full-sun case to be somewhat cooler than the full-eclipse case for the instrument (but not the spacecraft module) for a given season. The instrument is well-isolated from the sun, with shaded radiator surfaces, and the additional incident flux from the earth and albedo near the subsolar point appeared to offset the lower average solar flux, and array and spacecraft boundary temperatures.

The correlated model is not so effective in representing accurately the difference between beta $=90$ and beta $=0$. This difference has consistently been predicted as an in rease of $3-4^{\circ} \mathrm{C}$ at beta $=0$, but the flight data shows the $3=0$ temperatures to be equal to or slightly below use at beta $=90$. Power sensitivity is a bit more than $1^{\circ} \mathrm{C} /$ watt, and the slatform heater is about a watt less with lower voltage d. $L=:=0$, so the discrepancy means a 2-3 watt error in predicting powers absorbed from the environment. It is clear from longer-term trends that thermal effects of normal science-mission maneuvers are much larger than and probably obscure those of the environmental fluxes at nominal attitudes, and are beyond precise prediction with the models available.

The thermal model had not been correlated against transient conditions at the time of environmental test. However, accurate prediction by the model of transient conditions may be useful should orbital anomalies occur. A transient solution for mission days $1-3$ is shown in Figure 5 with measured data superimposed. In the

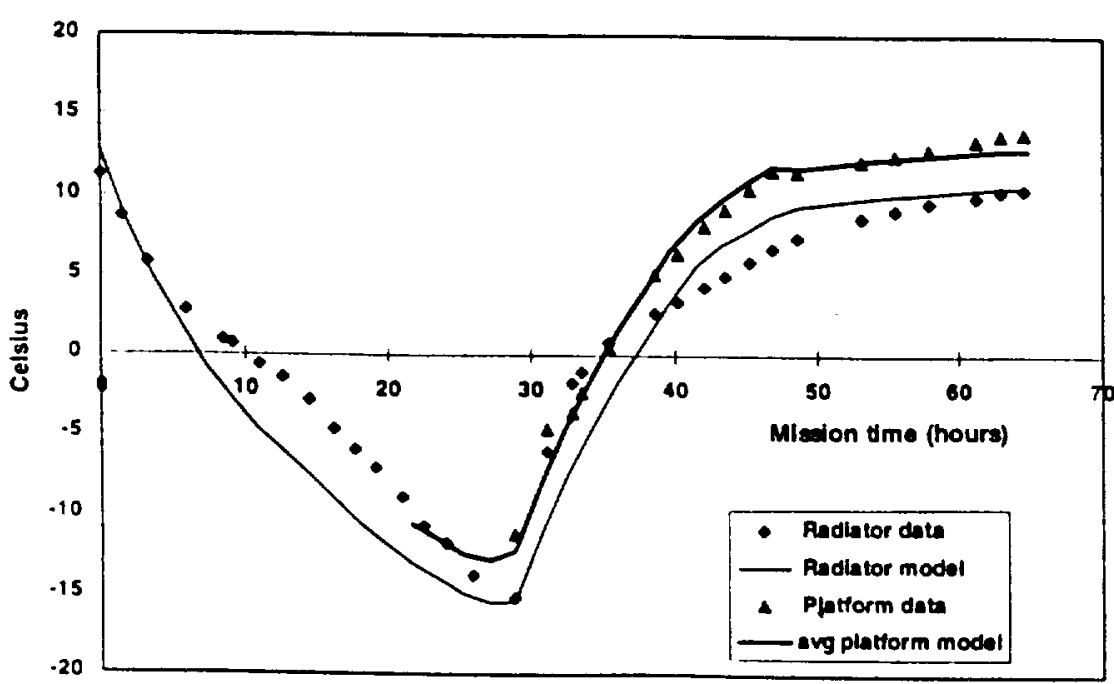

Figure 5:Model vs. Measured Flight Data - first 3 mission days cooldown phase the thermal model appears to predict somewhat faster response of the experiment than measured, but in fact this is probably caused by external environment changes, not captured by the model, on the external radiator location where the thermistor is located. There is nothing in the passive power-off cooldown that could cause the $S$-shape between hours 8-12 other than external influences. The model actually predicts a slightly longer time between turn-on of the two platform heaters at $10^{\circ} \mathrm{C}$ and $1{ }^{\circ} \mathrm{C}$ than was observed, and the full temperature drop to instrument power-on is accurately predicted.

After the instrument is powered and temperatures at the core of the experiment are available, the model transient response is quite accurate. Agreement of the model with data is generally within one Celsius, and turn-off of a platform heater at $10^{\circ} \mathrm{C}$ occurs at the correct time. The slope of the equilibration at the end of the three days is higher as measured, probably caused by initial startracker anomalies that resulted in unexpected highpower target-seek modes. We conclude that the thermal model's transient performance is better than could reasonably be expected, and that it is adequate for mission support in transient as well as steady-state conditions.

\section{PERFORMANCE OF THE COLD RECEIVER AND COMPOUND PARABOLIC RADIATOR SHADE}

The cold receiver at the focal plane has operated at 171$175^{\circ} \mathrm{K}$ during the early mission, considerably short of its $150^{\circ} \mathrm{K}$ design goal. This shortfall was compensated by the success of special efforts directed at thermal stability of critical electronics, so that science performance is largely unaffected.

Radiative capability of the three disc radiators is about $75 \%$ higher at observed mission conditions than at $150^{\circ} \mathrm{K}$. Parasitic loss into the cooled system, originally predicted at about one watt, is inferred to be nearly four watts. Much attention was directed during design to the mechanical and electrical interface of the cooled receiver, but the importance of mounting of the disc radiators themselves and the factors affecting performance of the MLI system were not sufficiently appreciated. Enclosing the receiver within the shell of the instrument, rather than allowing the outside of its MLI to view the colder space environment as originally planned, is likely responsible for most of the shortfall in performance. On the positive side, the flexible thermal links between receiver and radiators handled the higher losses with less than $1^{\circ} \mathrm{K}$ temperature drop, and the radiator shades performed very well, both in shading performance and in minimizing parasitic losses to the radiators within. 
In a near.earth orbit, shading a cold radiator from albedo and thermal radiation from the earth is of primary importance. To the authors' knowledge, SWAS is the first mission to use the Compound Parabolic Shade geometry, described earlier, to protect a cold radiator from parasitic incident environmental inputs. For a given entrance aperture and radiator size, the CPS axis can point closer to the earth's limb than a cone, and radiator parasitic heating from the shade interior is smaller. However, failure to maintain earth avoidance with a CPS produces larger heat gains on the radiator. Figure 6 shows effects of the sharpness of the CPS acceptance angle during an unintentional earth limb avoidance violation. The sudden temperature rise on the cold radiator is followed several minutes later by a rise in the cooled receiver. The change in receiver temperature is consistent with the energy absorbed during the time the earth's limb was within the acceptance angle. Almost a full day was needed for the cold radiator surfaces to reject at low temperatures the energy absorbed during the two-minute avoidance violation. Had a conical shade been used, the parasitic energy absorption would have begun at a larger angle from the limb, and the signature would not have been so dramatic. Performance of the cooler would have been degraded more gradually, and might not have been noticed so that it could be compensated for in the observing plan.

\section{THERMAL STABILITY AND SCIENCE DATA QUALITY}

Mission results have demonstrated the effectiveness of design emphasis on thermal stability and its direct contribution to science data quality. This arises because thermal variations are a primary source of systematic noise in an observation. For SWAS, long observing times are needed to distinguish faint signals from background; random background noise decreases as the square root of increased observing time. Systematic noise such as thermal drift in microwave amplifiers does not decrease with observing time, and can obscure faint signals. We took particular care in design to maximize thermal time constants of critical boxes by using mass distribution to advantage, adding isolation at mounts, and using MLI locally to block views to radiators where orbital and maneuvering thermal cycles would be largest. We also closed the radiator adjacent to the critical If amplifier module. Figure 7 shows temperatures on a typical mission day, comparing this module with a similar but non-critical electronics box. The orbital thermal cycle, evident in the electronics box, is not discernible in the amplifier module. This increased stability is a key to successful longer observations.

\section{LONG-TERM THERMAL TRENDS IN THE MISSION}

Orbital precession produced a few days of full-sun orbits in mid-March at beta $=70$ and again in May at beta $=89$, allowing a direct comparison of thermal conditions with those of the earliest full-sun orbits. Instrument temperatures were generally about $1.5^{\circ} \mathrm{C}$ lower in May than earlier near the winter solstice. Three beta $=0$
Figure 6: Cold Receiver and Radiator

Response to Briet Earth Vlew

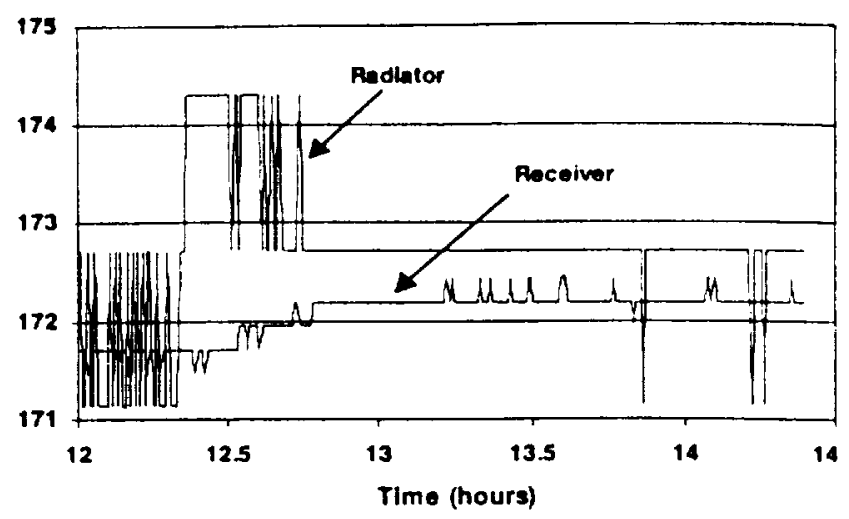

conditions from January to April showed a similar downward trend in instrument temperatures. Because degradation of surface properties is expected to cause higher temperatures, the lower temperatures show that 1500 sun hours had not caused significant degradation.

The SWAS instrument is well-isolated from most of the common long-term degradation effects of orbital duration. The silver-Teflon outer MLI layer is the only surface directly exposed to the sun, and its expected absorptance degradation, from less than 0.10 initially to 0.24 after three years, is greatly attenuated by the MLI. The white-painted radiator and CPS surfaces are not exposed to the sun and are little-affected by albedo. Less than a $5^{\circ} \mathrm{C}$ increase in overall instrument temperatures is predicted for end of mission life.

The spacecraft is also well-shielded from the sun, with primary long-term effects being degradation in solar array properties and in the launch adapter ring at the bottom. Spacecraft module temperatures were almost identical between the launch and May 1999 full-sun orbits. The body-mounted solar array peaked at $92^{\circ} \mathrm{C}$ in December vs. $87^{\circ} \mathrm{C}$ in May, a decrease consistent with the 5\%-lower solar constant. The spacecraft could rise as much as $10^{\circ} \mathrm{C}$ in the long term, mostly due to UV degradation of uncoated aluminum surfaces and the MLI outer layer (silver-Teflon) on the spacecraft sun side. Solar arrays will also heat up as they degrade, increasing backloading on spacecraft as well as instrument radiators.

Figure 7: Thermal Stability of Modules

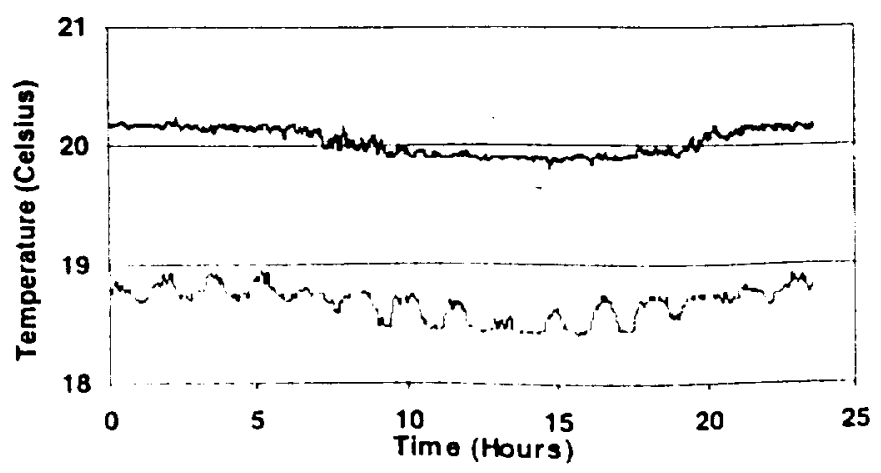


As we gain mission experience we are learning to incorporate thermal considerations into science larget selection. Elfects of allowing an earth-view violation were discussed earlier, and we have become more conservative in planned observing duration to avoid this. Solar absorption on the unprotected bottom aluminum adapter ring, also noted earlier, has distinct effects when observing targets at sun-satellite-target angles above 110 degrees. $A 7^{\circ} \mathrm{C}$ rise in spacecratt core temperatures and $2^{\circ} \mathrm{C}$ on the experiment platform were seen during a few days of the May full-sun orbits, when multiple targets having sun angles near 120 degrees were selected. Finally, a $1^{\circ} \mathrm{C}$ oscillation with a half-day period in a critical temperature was traced to observation of alternate targets having widely-different sun angles for periods of 6 hours each. This can be avoided without loss of science data by target-scheduling changes.

\section{CONCLUSIONS}

In retrospect, had a heater bus and modest control circuitry been incorporated as a contingency in the design, it could have been used locally for control of critical modules at little resource cost without ultimately restricting the range of the whole instrument. The incompatibility of a $20^{\circ} \mathrm{C}$ operating range using passive control with a large viewing aperture, large MLI areas, and three-axis pointing in near-earth orbit should have been evident.

Proper installation and careful design of MLI are equally necessary for successful thermal performance of a lowpower instrument. (Redesign of the SWAS instrument MLI reduced instrument heat losses over 50\%.) Allowing for thermal contraction of outer layers (up to $1 \%$ for spacefacing surfaces) and for circumferential differences in convex blarikets ensures that thickness compression is avoided on-mission; this is particularly critical when MLI is assembled on a flat table. Teflon requires special care because it contracts more than Kapton when cold.

Heaters with power that is insufficient to cause cycling of control thermostats can provide thermal control margin where temperature stability is needed and switched power services are not available.

Survival heater set-point selection should allow for operational considerations such as temperature rates of change, frequency of ground station contacts, and available telemetry.

Quick-running reduced models available during launch operations are useful for evaluating non-nominal attitudes and timelines, especially for evaluation of temperature differences between telemetered points and critical areas.

Models and environmental tests often do not anticipate all the variability in a science mission. Unexpected effects can appear well into the mission as the science team extends the capability of the equipment and develops new observing schemes. Long-term support by engineering staff can be a direct contribution to refinement and improvement of science data quality and conduct of the mission.

\section{ACKNOWLEDGEMENTS}

The authors would like to thank Bob Poley and Gretchen Antoniak of Ball Aerospace, and Ted Michalek of NASA Goddard, for their contributions to the SWAS instrument thermal design.

\section{REFERENCES AND NOTES}

[1]Welford, W.T. and Roland Winston, The Optics of Non-imaging Concentrators, Academic Press, New York, 1978

[2]Boyd, D.A. and Wes Ousley, The Submillimeter-Wave Astronomy Satellite: Instrument Thermal Design and Verification, SAE Technical Paper No. 961493, 1996

Gore-tex is a trademark of W.L. Gore \& Associates, Inc. Kapton, Teflon and Mylar are trademarks of DuPont Inc. 